\title{
Phase-Based Adaptive Estimation of Magnitude-Squared Coherence Between Turbofan Internal Sensors and Far-Field Microphone Signals
}

\author{
JEFFREY HILTON MILES *† \\ NASA John H. Glenn Research Center at Lewis Field, Cleveland, OH 44135
}

$\ddagger$

\begin{abstract}
A cross-power spectrum phase based adaptive technique is discussed which iteratively determines the time delay between two digitized signals that are coherent. The adaptive delay algorithm belongs to a class of algorithms that identifies a minimum of a pattern matching function. The algorithm uses a gradient technique to find the value of the adaptive delay that minimizes a cost function based in part on the slope of a linear function that fits the measured cross power spectrum phase and in part on the standard error of the curve fit. This procedure is applied to data from a Honeywell TECH977 static-engine test. Data was obtained using a combustor probe, two turbine exit probes, and far-field microphones. Signals from this instrumentation are used estimate the post-combustion residence time in the combustor. Comparison with previous studies of the post-combustion residence time validates this approach. In addition, the procedure removes the bias due to misalignment of signals in the calculation of coherence which is a first step in applying array processing methods to the magnitude squared coherence data. The procedure also provides an estimate of the cross-spectrum phase-offset.
\end{abstract}

\section{Nomenclature}

a Zero intercept of two parameter phase function $\phi_{k m}^{\prime}(f)$

$B_{e} \quad$ Bandwidth resolution, $B_{e}=\Delta f=1 / T_{d}=r_{s} / N, 16 \mathrm{~Hz}$

$b_{k m} \quad$ Slope of cross-spectrum phase curve

$C_{o}, C_{\max }, C_{\min }, C$ Cost functions

$D_{\ell m}$ Propagation time from turbine exit to far-field microphone

$D_{k \ell} \quad$ Post combustion residence time in the combustor, $D_{k \ell}=D_{k m}-D_{\ell m}$

$D_{k m}$ Propagation from combustor to far-field microphone

$E[$ ] Effective Value of $E[$ ]

$f \quad$ Frequency, $\mathrm{Hz}$

$f_{u} \quad$ Upper frequency limit, $f_{u}=1 / 2 \Delta t=r_{s} / 2, \mathrm{~Hz}(32768 \mathrm{~Hz})$

$F_{2} \quad$ Weight function

$\hat{G}_{\ell \ell}(f)$ Estimated output signal one-sided auto spectral density function

$\hat{G}_{k k}(f)$ Estimated input signal one-sided auto spectral density function

$\hat{G}_{k m}(f), \hat{G}_{\ell m}(f)$ Estimated one-sided cross-spectral density function

$J \quad$ Cost function

$N \quad$ Segment length, number of samples per segment, $N=r_{s} T_{d},(4096)$

*Aerospace Engineer, Acoustics Branch,21000 Brookpark Road, Cleveland, Ohio, 44135

${ }^{\dagger}$ AIAA Associate Fellow

$\ddagger$ November 22, $2014 \mathrm{H}: \backslash \operatorname{mm140529}$-sigalign \mm140529.tex

1 of 19

American Institute of Aeronautics and Astronautics 
$n_{k \ell} \quad$ Number of points used in two parameter curve fit

$n_{o} \quad$ Number of segments/blocks with 50 percent overlap, $n_{o}=2 B_{e} T_{\text {total }} \approx 2240$

$n_{s} \quad$ Number of disjoint (independent) segments used in spectra estimates, $n_{s}=B_{e} T_{\text {total }} \approx 1120$

$r(t) \quad$ Acoustic signal

$R_{k m}(\tau)$ Cross-correlation function

$r_{s} \quad$ Sample rate, samples/s (65536)

$S \quad$ Cost function sums

$s(t) \quad$ Acoustic signal

$\left.S_{[}\right] \quad$ Summation function over [ ]

$t \quad$ Time variable

$T_{\text {total }} \quad$ Total record length, $T_{\text {total }}=n_{s} T_{d}, \mathrm{~s}(\approx 70 \mathrm{~s})$

$T_{d} \quad$ Record length of segment, $T_{d}=N / r_{s}, 0.0625 \mathrm{~s}$

$W_{k} \quad$ Weight factor

$x_{j} \quad$ Frequency $f_{j}$

$y_{j} \quad$ Measured cross-spectrum phase $\phi_{k m}^{m}$

Subscripts

1 Combustion location

2 Turbine exit location

3 microphone location

$\ell \quad$ Location is turbine

$k \quad$ Location is combustor

$m \quad$ Location is far-field microphone

$x \quad$ Input signal

y Output signal

Symbols

[] Mean value of [ $\left.{ }^{-}\right]$.

$<>\quad$ Angular brackets denote long time averages.

$\beta_{k m} \quad$ Slope of two parameter phase function $\phi_{k m}^{\prime}(f)$

$\Delta t \quad$ Sampling interval, $\Delta t=1 / r_{s} \quad \mathrm{~s}$

$\epsilon \quad$ Residual

$\hat{\gamma}_{x, y}^{2}(f)$ Estimated magnitude-squared coherence (MSC) function

$\hat{\gamma}_{x_{n}, y_{n}}^{2}\left(n_{s}\right)$ MSC threshold function

$\left[{ }^{0}\right]$ Estimate of [ ]

$\Im$ Imaginary part

$\nu \quad$ weight value

$\omega \quad$ Angular frequency, $\omega=2 \pi f$

$\phi \quad$ Angle variable

$\phi_{k m}^{\prime}(f)$ Two parameter linear curve fit function

$\phi_{0} \quad$ Zero intercept of two parameter phase function $\phi_{k m}^{\prime}(f)$

$\phi_{k m}(f)$ Cross-spectrum phase function

$\psi \quad$ Weight factor

$\Re \quad$ Real part

$\sigma_{k m}^{2} \quad$ Residual variance

$\sigma_{k m} \quad$ Standard error

$\tau \quad$ Time displacement

Int Integer part

$m \quad$ Measured

\section{Introduction}

The technique introduced in this paper is an adaptive estimation process which synchronizes two signals correlated over a frequency range using a time domain iterative algorithm. This procedure will enable 
removal of the bias due to misalignment of signals in the calculation of coherence and will estimate more accurately the cross-power spectrum phase-offset.

The algorithm uses a cost function and a gradient approximation to continually update the time delay estimate until the process has minimized the cost function. The cost function is based on a linear curve fit to the unwrapped phase of the cross-spectrum between the two signals over the frequency range of interest. The linear curve fit assumes the existence of a cross-spectrum phase-offset and does not assume the linear fit has a zero intercept at zero frequency. The iterative technique used is a steepest-descent gradient technique and thus requires that the cost function is unimodal.

\section{A. Relevance}

Low frequency noise generated in the turbofan engine core can make a significant contribution to the overall noise signature in the aft direction at the low power settings which are used on an airport flight approach trajectory. This type of low frequency noise may become even more of a problem for future aircraft. Two possible low frequency noise sources are direct and indirect combustion noise. The source of combustion noise attributed to the unsteady pressures produced by the unsteady combustion process that propagate through the turbine to the far field is called the direct combustion noise source. The other source of turbofan engine combustion noise is known as the indirect mechanism in which the noise is generated in the turbine by the interaction of entropy fluctuations, which also originate from the unsteady combustion process, as they propagate through regions characterized by mean flow velocity or pressure gradients in the turbine stages. This indirect noise source was studied using analytical models by Ffowcs Williams and Howe, ${ }^{1}$ Pickett, ${ }^{2}$ Marble and Candel,${ }^{3}$ Cumpsty and Marble, ${ }^{4,5}$ Cumpsty, ${ }^{6}$ Gliebe et al. ${ }^{7}$ Mani $_{1}^{8}$ Bodony ${ }^{9}$ and Leyko ${ }^{10}$.

The net travel time of the indirect combustion noise signal from the combustor to the turbine exit and the far field is longer than the direct one since the travel velocity of the entropy fluctuations to the turbine is the flow velocity in the combustor. This flow velocity is a small fraction of the speed of sound. Miles et al. ${ }^{11,12}$ has shown the pressure and entropy should be in phase in the combustor. Consequently, one might expect that the pressure signal from an indirect combustion noise source would be delayed relative to a pressure signal from a direct combustion noise source since an indirect combustion noise signal does not travel with the speed of an acoustic wave until it interacts with the turbine. Miles ${ }^{13-15}$ with data from the Honeywell TECH977 engine test program ${ }^{16}$ shows that the cross-spectra and correlation function between a combustor sensor and far-field microphones are tools that provides a way to separate low frequency direct and indirect coherent combustion noise due to this travel delay time.

\section{B. Adaptive Estimation}

Etter and Stearns ${ }^{17}$ describe an autocorrelation function based adaptive estimation process for time delays for possible use with sonar or radar signals. A similar procedure is outlined by Carter ${ }^{18}$ for passive sonar signal processing which uses a cross-correlation function. The generalized correlation method for estimation of time delay that uses a pair of prefilters before a cross-correlation process has been discussed by Knapp and Carter ${ }^{19}$ and Azaria and Hertz. ${ }^{20}$

These methods are early versions of modern signal-processing algorithms now widely used in medical ultrasound, speech processing and other applications that can be more generally described as the identification of a minimum (or maximum, depending on the particular algorithm used) of a pattern matching function. ${ }^{21-23}$ A great many pattern matching algorithms are used. Many are reviewed by Jacovitti and Scarano ${ }^{21}$ and Viola and Walker. ${ }^{23}$ While some cost functions use cross correlation, in many applications other signal matching measures have been developed for applications requiring intensive or real-time processing.

\section{Present Technique}

The adaptive estimation process used herein estimates the time delay with a cost function that is based on the unwrapped cross-spectrum phase between two sensors over a preselected frequency range. The cost function is based on the slope of the regression line and the standard deviation of the linear curve fit to the phase. It is assumed that the time series overlap for a time delay that minimizes this cost function. The procedure at each iterative step determines the value of the cost function and continually realigns the time series by varying the time lag until the cost function has been minimized and thereby effectively has reduced 
the slope to zero. The frequency interval is preselected so that the magnitude-squared coherence between signals is not too small in the selected range. A non-adaptive time delay estimation method that uses a linear fit with a zero intercept at zero frequency to unwrap cross-spectrum phase is described by Piersol. ${ }^{24}$ In contrast, the method used herein does not require the measured phase shift sequence to pass through the origin which is an assumption used by Piersol ${ }^{24}$ and other investigators. ${ }^{25-27}$ Since the method uses only a selected portion of the calculated phase, it is called a generalized unwrapped phase method. The same results could be achieved at more computational expense by using a pair of pre-filters to select the frequency range of interest as is done by Knapp and Carter. ${ }^{19}$ Other non-adaptive methods that estimate a time delay using the cross-spectrum phase are available. ${ }^{25-27}$

This adaptive estimation method of calculating the time delay is applied to time series measurements in the combustor, at the turbine exit, and at select far-field microphones from the Honeywell TECH977 test program. ${ }^{16}$ In order to calculate the post-combustion residence time in the combustor i.e. the time delay of the indirect combustion noise in the combustor, the propagation time from the combustor probe and the turbine exit probe to a far-field microphone are directly estimated by the procedure and subtracted. This is the first application of time delay estimation using a phase based adaptive pattern matching function to measure the post-combustion (post-flame) residence time in a turbofan engine combustor using signals measured at the turbine exit with signals from a combustor sensor probe and far field microphones.

\section{Engine test data}

The NASA/Honeywell static engine test program was conducted at Honeywell's San Tan outdoor acoustic test facility using a Honeywell TECH977 engine (Fig. 1) and the results are described in a report by Weir. ${ }^{16}$ The dual-spool, turbofan engine has a direct drive, wide chord fan connected by a long shaft to the low-pressure turbine spool and a high-pressure compressor connected by a concentric short shaft to the turbine high-pressure spool. The fan diameter is about $0.87 \mathrm{~m}$. The combustor design is a straight-throughflow annular geometry with 16 fuel nozzles and 2 igniters. Data obtained for one configuration in the test program is analyzed in this paper. The engine-internal instrumentation in this configuration included hightemperature pressure sensors with air cooling in a combustor igniter port identified herein as CIP1 and at the turbine exit sensors identified as T551 and T552. Pressure time histories from these internal sensors and far-field microphones are used herein.

The data acquisition system had a sampling rate of $65536 \mathrm{~Hz}$ and a sampling duration of roughly $70 \mathrm{~s}$. The spectra were calculated using a 50 percent overlap. This permitted data reduction using $\approx 1120$ non-overlapped ensemble averages or with a 50 percent overlap $\approx 2240$ overlapped ensemble averages at a bandwidth resolution of $16 \mathrm{~Hz}$. Further signal estimation parameters are shown in Table 1.

The engine condition power settings discussed are 48 and 54 percent of the Max Power setting. Microphone locations used are at $90^{\circ}, 110^{\circ}, 130^{\circ}$, and $160^{\circ}$ measured from the inlet. The test was conducted when the air temperature was about $9^{\circ} \mathrm{C}\left(48.2^{\circ} \mathrm{F}\right)$ and the microphone radius was $30.48 \mathrm{~m}$. The engine was at a height of $3.049 \mathrm{~m}$.

As discussed, ${ }^{13,28}$ the size of the magnitude-squared coherence between the combustor sensor and a far-field microphone identifies the spectral region of importance for indirect combustion noise as being in the $0-200 \mathrm{~Hz}$ frequency range. Consequently, herein the cross-spectral phase measurements between engineinternal sensors and a far-field microphone only over the frequency range $16-208 \mathrm{~Hz}$ is used to estimate the propagation time to a far field microphone.

\section{Theory}

\section{A. Procedure}

The far-field microphones receives noise from the turbofan, jet, and core. The procedure characterizes the core noise as indirect combustor noise and turbine-exit coherent broadband noise. Furthermore, the method estimates the propagation time from source to far-field microphone. A simple mathematical model for the propagation time measurements is

$$
\begin{aligned}
& r_{k}(t)=s_{1}\left(t-D_{k m}\right)+n_{1}(t) \text { Combustor Sensor } \\
& r_{\ell}(t)=s_{2}\left(t-D_{\ell m}\right)+n_{2}(t) \text { Turbine Sensor }
\end{aligned}
$$

$$
4 \text { of } 19
$$




$$
r_{m}(t)=s_{3}(t)+n_{3}(t) \text { Microphone }
$$

where the signal at station $k$ from the combustion pressure sensor is delayed by the propagation time delay, $D_{k m}$, the turbine pressure sensor signal at location $\ell$ is delayed by the propagation time delay, $D_{\ell m}$, the signal at station $m$ is from the far-field microphone.

The signal at station $k$ is from the combustor and the acoustic pressure signal $s_{1}(t)$ is assumed to be related to an entropy or temperature disturbance. This disturbance moves at the combustor flow velocity to the turbine where it interacts with the pressure field creating indirect combustion noise which reaches a microphone after a total travel time, $D_{k m}$. The signal at station $\ell$ is from the turbine. The acoustic pressure signal $s_{2}(t)$ is from the turbine exit coherent broadband noise which reaches a microphone after a total travel time, $D_{\ell m}$. In either case the procedure to finding the time delay is identical and is described in the algorithm section. The signals at station $k, \ell$ and $m$ are contaminated by independent, Gaussian, and stationary noise terms, $n_{1}(t), n_{2}(t)$, and $n_{3}(t)$, which are uncorrelated with $s_{1}(t), s_{2}(t)$, and $s_{3}(t)$ and each other

The cross-correlation function between the combustor signal and a far-field microphone is given by

$$
R_{k m}(\tau)=E\left[r_{k}(t) r_{m}(t+\tau)\right]=R_{s_{1} s_{3}}\left(\tau-D_{k m}\right)
$$

where $R_{s_{1} s_{3}}\left(\tau-D_{k m}\right)$ is the autocorrelation function with a peak at $\tau=D_{k m}$.

In the frequency domain, the Fourier transform of equation (4) is

$$
G_{k m}(f)=G_{s_{1} s_{3}}(f) \exp \left(-i 2 \pi f D_{k m}\right)
$$

where $G_{s_{1} s_{3}}$ is the one-sided cross-spectrum and the time delay $D_{k m}$ appears in the cross spectrum as a phase function

$$
\phi_{k m}(f)=2 \pi f D_{k m}=\omega D_{k m}
$$

where $\omega=2 \pi f$. The magnitude-squared coherence (MSC) is defined by

$$
\gamma_{k m}^{2}(f)=\frac{\left|G_{k m}(f)\right|^{2}}{\left|G_{k k}(f)\right|\left|G_{m m}(f)\right|}
$$

by

The cross-correlation function between the turbine probe signal and a far-field microphone is given

$$
R_{\ell m}(\tau)=E\left[r_{\ell}(t) r_{m}(t+\tau)\right]=R_{s_{2} s_{3}}\left(\tau-D_{\ell m}\right)
$$

where $R_{s_{2} s_{3}}\left(\tau-D_{\ell m}\right)$ is the autocorrelation function with a peak at $\tau=D_{\ell m}$.

In the frequency domain, the Fourier transform of equation (8) is

$$
G_{\ell m}(f)=G_{s_{2} s_{3}}(f) \exp \left(-i 2 \pi f D_{\ell m}\right)
$$

where $G_{s_{1} s_{3}}$ is the one-sided cross-spectrum and the time delay $D_{\ell m}$ appears in the cross spectrum as a phase function.

$$
\phi_{\ell m}(f)=2 \pi f D_{\ell m}=\omega D_{\ell m}
$$

The magnitude-squared coherence (MSC) is defined by

$$
\gamma_{\ell m}^{2}(f)=\frac{\left|G_{\ell m}(f)\right|^{2}}{\left|G_{\ell \ell}(f)\right|\left|G_{m m}(f)\right|}
$$

Then $D_{k \ell}=D_{k m}-D_{\ell m}$. With a small error since the acoustic propagation speed are high and the distances involved are small, the indirect combustion noise is dealt with as if it all begins propagating acoustically at the turbine exit and then it propagates to a microphone in the same manner as the direct combustion noise. 


\section{B. Signal Processing Method .}

All the spectra and cross-spectra are estimated using Welch's nonparametric method which is based on averaging multiple windowed periodograms using overlapping time sequences. ${ }^{29-33}$ Using these spectra and cross spectra, the magnitude-squared coherence (MSC) is calculated to measure the similarity of the amplitude variations at particular frequencies. ${ }^{33}$ In some sections, the ${ }^{\wedge}$ accent will be used to denote the statistical basis of a variable and that it is based on a finite time series.

\section{Accuracy of Estimates}

We consider the cross spectrum magnitude estimate, $\hat{G}_{k m}(f)$, and phase estimate, $\hat{\phi}_{k m}(f)$, computed from Fourier transforms of signals $r_{1}(t)$ and $r_{2}(t)$ calculated for a sample length $T_{\text {total }}$ divided into $n_{s}$ independent samples using a periodogram procedure. The estimated cross-spectrum $\hat{G}_{k m}$ is a complex number. The estimated cross-spectrum phase $\hat{\phi}_{k m}$ is given by

$$
\hat{\phi}_{k m}=\arctan \left[\frac{\Im \hat{G}_{k m}}{\Re \hat{G}_{k m}}\right]
$$

The spectral estimates are statistically independent of one another and have a standard deviation discussed by Piersol $^{24}$, derived by Bendat, ${ }^{34}$ and also presented in a book by Bendat and Piersol ${ }^{35}$ approximately given by

$$
\begin{aligned}
\sigma\left[\left|\hat{G}_{k m}\left(f_{i}\right)\right|\right] & \approx \frac{\left|G_{k m}\left(f_{i}\right)\right|}{\sqrt{n_{s} \gamma_{k m}^{2}\left(f_{i}\right)}} \\
\sigma\left[\hat{\phi}_{k m}\left(f_{i}\right)\right] & \approx \arcsin \left[\frac{1-\gamma_{k m}^{2}\left(f_{i}\right)}{2 n_{s} \gamma_{k m}^{2}\left(f_{i}\right)}\right]^{1 / 2}
\end{aligned}
$$

For small phase errors when $\sin \sigma=\sigma$

$$
\sigma\left[\hat{\phi}_{k m}\left(f_{i}\right)\right] \approx\left[\frac{1-\gamma_{k m}^{2}\left(f_{i}\right)}{2 n_{s} \gamma_{k m}^{2}\left(f_{i}\right)}\right]^{1 / 2}
$$

It can be seen from Eqs (14)-(15) that the error in the estimated phase angle becomes infinitely large as the MSC tends to zero. This is the reason why only a limited frequency interval is used in the generalized adaptive phase method.

\section{True MSC based on the Adaptive Estimation Procedure}

To a first order approximation the computed/estimated unaligned and aligned MSC based on the far-field microphones and probes in the combustor and at the turbine exit are related by

$$
\begin{aligned}
& \left.\left.\hat{\gamma}_{k m}^{2}(f)\right|_{\text {unaligned }} \approx\left(1-\frac{D_{k m}}{T_{d}}\right)^{2} \gamma_{k m}^{2}(f)\right|_{\text {aligned }} \\
& \left.\left.\hat{\gamma} \ell m_{\ell m}^{2}(f)\right|_{\text {unaligned }} \approx\left(1-\frac{D_{\ell m}}{T_{d}}\right)^{2} \gamma_{\ell m}^{2}(f)\right|_{\text {aligned }}
\end{aligned}
$$

if $D_{k m}$ and $D_{\ell m}$ are less than $T_{d}$ (Bendat $^{34}$ and Bedat and Piersol ${ }^{35}$ ).

To increase the number of blocks of data sampled to get better statistics the bandwidth resolution, $B_{e}$, used is $16 \mathrm{~Hz}$ and the segment length, $T_{d}$, of the signal is then $62.5 \mathrm{~ms}$. However, the time delay from the engine probes to the far field-microphone, $D_{k m}$ and $D_{\ell m}$ is between $70 \mathrm{~m} \mathrm{~s}$ and $90 \mathrm{~ms}$. Consequently, the alignment procedure must be used since using non-aligned measurements one would only measure the MSC threshold which is small. An order of magnitude estimate of the threshold for a 95 percent confidence interval and $n_{s}=1120$ independent blocks of data is (Miles ${ }^{36,37}$ )

$$
\hat{\gamma}_{x_{n}, y_{n}}^{2}\left(n_{s}\right)=1-(1-0.95)^{1 /\left(n_{s}-1\right)}=2.67 \times 10^{-3} .
$$

\section{6 of 19}


By aligning the measurements and removing any time delay the true MSC can be measured. The generalized unwrapped phase adaptive estimation procedure does need an initial condition for $D_{k m}$ and $D_{\ell m}$. An estimated value of $70 \mathrm{~m} \mathrm{~s}$ to $90 \mathrm{~ms}$ based on the acoustic speed of sound and the $100 \mathrm{ft}$ microphone radius is sufficient for this purpose.

\section{E. Algorithm}

The current algorithm identifies the dominant noise source at the turbine exit as either direct or indirect combustion noise over a certain frequency range. It does this by calculating the difference in delay time, $D_{k \ell}$, for a signal from the combustor to reach a far-field microphone and a signal from the turbine exit to reach the same far-field microphone. If both time delays are about the same, the dominant noise source is direct combustion noise. If the time delay difference is in the region of 3 to $4 \mathrm{~ms}$ the dominant noise source is indirect combustion noise.

The general idea is that the noise from the turbine is acoustic while the noise in the combustor has a spectrum related to the noise created by entropy waves interacting with the pressure field in the turbine. This interaction produces noise after a time delay, $D_{k \ell}$, related to the convection velocity in the combustor and the distance from the flame zone to the turbine. This post-combustion (post-flame) time delay, $D_{k \ell} \ell$, shows up in the phase of the cross-spectra of the combustion noise signal and a far-field microphone signal.

The procedure used is to first evaluate the time delay from the combustor and far-field microphone cross spectrum, $D_{k m}$. This is done by iteratively and optimally resetting the origin of the time history of the far-field microphone signal and at each step calculating the pressure cross-spectrum phase between the combustor probe signal and the far field microphone signal until the slope of the phase plot curve is zero. This time delay is the acoustic travel time, $D_{\ell m}$, plus the post-combustion (post-flame) time delay, $D_{k} \ell$. Next the same method is used to evaluate the time delay from the turbine exit probe signal and the same far-field microphone, $D_{\ell m}$. Again this is done by iteratively and optimally resetting the origin of the time history of the far-field microphone signal and at each step calculating the pressure cross-spectrum phase between the turbine exit probe signal and the far field microphone signal until the slope of the phase plot curve is zero. This provides an estimate of the acoustic travel time from the turbine exit to the same microphone, $D_{\ell} m$ Consequently, the difference in these actual time delays is the post-combustion (post-flame) time delay, $D_{k \ell}$. The indirect combustion noise is treated as if it all begins propagating acoustically at the turbine exit and then it propagates to each microphone in the same manner as the direct combustion noise. The resolution is limited by the sampling rate. For this test program the sampling rate is $65,536 \mathrm{samples} / \mathrm{s}$. Consequently, the value is known within $0.015 \mathrm{~ms}(\approx 1000 / 65,536 \mathrm{~ms})$.

In order to estimate either time delay, $D_{k \ell}$, a pattern matching function based on a regression line fit to the measured cross-spectrum phase $\hat{\phi}_{k} \ell$ will be used. However, the regression line fit is not forced to have a zero intercept because 1) the pressure sensors are not the same; 2) the pressure sensors are not phase matched; 3 ) the phase measured at $f=0$ will not be used in the time delay calculations; and 4) while a large number of independent samples are used and the MSC is greater than 0.01, the phase is calculated with uncertainty fluctuations. In addition, the MSC plots show the phase related to the indirect combustion noise is in the frequency range from $16-208 \mathrm{~Hz} .{ }^{13,28}$ Consequently, only phase function points in that frequency range are used.

\section{Estimation of slope of cross-spectra phase and cost function}

Instead of using a simple linear regression model with a zero intercept at $f=0$, a simple two parameter linear regression model is used. The procedure will be described using the combustor microphone and the far-field microphone.

$$
\phi_{k m}^{\prime}(f)=a+\beta_{k m} f+\epsilon
$$

The parameters are estimated using the least square method that minimizes the sum of squares of the vertical distances $\left.\left(S=\sum_{j}\left(\hat{\phi}_{k m}^{m}\left(f_{j}\right)-a-\beta_{k m} f_{j}\right)^{2}\right)\right)^{38-41}$ Let $x_{j}=f_{j}$ and $y_{j}=\hat{\phi}_{k m}^{m}\left(f_{j}\right)$ where the time delay is $D_{k m}=\beta_{k m} /(2 \pi), a=\phi_{0}$ and $\epsilon$ is an error due to uncertainty fluctuations.

Then 


$$
\begin{aligned}
S_{x} & =\sum_{j} x_{j} \\
S_{y} & =\sum_{j} y_{j} \\
S_{x y} & =\sum_{j} x_{j} y_{j} \\
\bar{x} & =\frac{S_{x}}{n_{k \ell}} \\
\bar{y} & =\frac{S_{y}}{n_{k \ell}} \\
S_{x^{2}} & =\sum_{j}\left(x_{j}-\bar{x}\right)^{2} \\
S_{y^{2}} & =\sum_{j}\left(y_{j}-\bar{y}\right)^{2} \\
\beta_{k \ell} & =\frac{\left(S_{x y}-n_{k \ell} \bar{x} \bar{y}\right)}{S_{x^{2}}} \\
& =\frac{\sum_{j}\left(y_{j}-\bar{y}\right)\left(x_{j}-\bar{x}\right)}{S_{x^{2}}} \\
a & =\bar{y}-\beta_{k \ell}
\end{aligned}
$$

The residual variance is

$$
\sigma_{k m}^{2}=\frac{\left.\sum_{j}\left(\hat{\phi}_{k m}\left(f_{j}\right)-a-\beta_{k m} f_{j}\right)^{2}\right)}{n_{k m}-2}
$$

The standard error of the measurement is simply $\sigma_{k m}$.

The cost function used to adjust the time delay $D_{k \ell}$ to minimize the slope of the linear curve fit to the thirteen phase measurements of interest and reduce the standard error is

$$
C_{0}=\left(\beta_{k m}+\nu \sigma_{k m}\right)^{2}
$$

The parameter $\nu$ is selected to be about $1.0 E-04$ so that if a value of $\sigma_{k m}$ is 20 , the value of $\sigma_{k m}$ will not have an impact on the cost function until the slope, $\beta_{k m}$, has been reduced to 1.E-03. In order to prevent the search procedure from diverging two additional cost functions were defined.

$$
\begin{aligned}
& C_{\text {max }}=F_{2}\left(D_{k m}-D_{\max }\right)^{2} \text { if } D_{k m}>D_{\max } \\
& C_{\min }=F_{2}\left(D_{\min }-D_{k m}\right)^{2} \text { if } D_{k m}<D_{\min }
\end{aligned}
$$

where $D_{\max }$ is some convenient number like $100 \mathrm{~ms}$ and $D_{\min }$ is some convenient number like $70 \mathrm{~ms}$ and $F_{2}$ is has some value like 10 or 100 . These values serve to reduce the search region as needed.

The total cost function, $\mathrm{C}$, is

$$
C=C_{0}+C_{\max }+C_{\min }
$$

\section{Parameter evaluation procedure}

The search technique used in this study is described by Powell ${ }^{42}$ and a Fortran computer code for this algorithm is given by Kuester. ${ }^{43}$ The search program evaluates. a new cost function with each iterative selection of $D_{k m}$. The signal $r_{k}$ is measured at the combustor entrance at the same time as the signal $r_{\ell}$ is measured at the turbine exit and as each microphone signal is measured The subroutine that calculates the cost function time shifts the signal from the far-field microphone, $r_{k}$, by an amount $n_{k m}$ using Fortran code 


$$
\begin{aligned}
& \text { Ln993: do } j=0, \mathrm{Lx} \\
& \begin{aligned}
r_{k}(j)= & r_{k}\left(j+n_{k m}\right) \\
& \text { enddo } \operatorname{Ln} 993
\end{aligned}
\end{aligned}
$$

where if the dominant low frequency noise source is indirect combustion noise, we let $n_{k m}=\operatorname{Int}\left(r_{s} \times D_{k m}\right)$ for the combustor signal case and $n_{\ell m}=\operatorname{Int}\left(r_{s} \times x D_{\ell m}\right)$ for the turbine signal case. The actual value of $D_{\ell m}$ depends on the acoustic propagation velocity and distance along the paths from the turbine to the nozzle and from the nozzle through the jet at the nozzle exit and then to a microphone on the ground. The actual value of $D_{k m}$ depends on $D_{\ell m}$ and the velocity of the gas flow in the combustor and the distance in the combustor from the flame zone to the turbine. The cross-spectrum between the signals $r_{k}$ and $r_{m}$ is calculated. Next, a new cost function is calculated and the iterative procedure continues until it has converged.

The resulting phase plot of $\left.\phi_{k m}\right|_{\text {zero slope }}$ over the frequency range from $16 \mathrm{~Hz}$ to $208 \mathrm{~Hz}$ where the MSC is $\geq 0.1$ after convergence will show a a set of points moving above and below a horizontal line in a random fashion due to uncertainties caused by noise where

$$
\left.\phi_{k m}\right|_{\text {zero slope }} \approx \arctan \left[\frac{\Im\left(\left.G_{s_{1} s 3}\right|_{\text {zero slope }}(f) \exp \left(-\left.i \phi_{s_{1} s 3}\right|_{\text {zero slope }}(f)\right)\right)}{\Re\left(\left.G_{s_{1} s 3}\right|_{\text {zero slope }}(f) \exp \left(-\left.i \phi_{s_{1} s 3}\right|_{\text {zero slope }}(f)\right)\right)}\right]
$$

\section{Results}

\section{A. Post-combustion residence time in the combustor}

The post-combustion (post-flame) residence times in the combustor, $D_{k \ell}$, as determined for the 48 and 54 per cent of maximum power operating condition cases is shown in Tables 2 and 3 . These tables shows the propagation time for the turbine exit sensors is in the range of 86 to $94 \mathrm{~ms}$ and the indirect combustion noise signal takes 3.6 to $4.03 \mathrm{~ms}$ longer. Each table shows the actual number of counts and time in ms required to shift the far-field pressure signal to make it align with the combustion sensor signal.

\section{B. Analysis of distribution of post-combustion residence times in the combustor}

The distribution of the estimated post-combustion residence times in the combustor were analyzed using histogram density plots using the methods described by Fox ${ }^{44}$ and by Fox and Weisberg. ${ }^{45}$ The histogram is constructed so that the areas of the histogram bars sum to unity. Kernel density estimates are shown for the normal bandwidth (the line is of double thickness) and half the normal bandwidth (the line is of normal thickness). Also shown at the bottom of the plot are the location of the actual estimates as a scatterplot (or "rugplot") using a vertical bar | as the plotting symbol. A histogram of the post combustion residence times in the combustor at the 48 percent maximum power case is shown in Fig 2. Also shown is the mean value of the post-combustion time delay in the combustor at $\tau_{\text {mean }}=3.960 \mathrm{~ms}$. Using an alternative procedure with signals from only the combustor probe and turbine exit probes Miles ${ }^{46}$ calculated a time delay of $\tau_{C I P 1, T 551}=3.937 \mathrm{~ms}$ and a time delay of $\tau_{C I P 1, T 552}=4.028 \mathrm{~ms}$. These are also shown as vertical lines and are in close agreement with the values calculated using far-field microphones.

A histogram of the post combustion residence times in the combustor at the 54 percent maximum power case is shown in Fig 3. Also shown is the mean value of the post-combustion time delay in the combustor at $\tau_{\text {mean }}=3.791 \mathrm{~ms}$. Using the alternative procedure with signals from only the combustor probe and the turbine exit probes Miles ${ }^{46}$ calculated a time delay of $\tau_{C 1 P 1, T 551}=3.753 \mathrm{~ms}$ and a time delay of $\tau_{C I P 1, T 552}=3.921 \mathrm{~ms}$. These are also shown as vertical lines and are in close agreement with the values calculated using far-field microphones. The values obtained by Miles ${ }^{13-15,28,47}$ using other methods are comparable to these.

\section{Phase and MSC measurements at 48 percent maximum power}

Aligned MSC and cross-spectrum phase measurements between the combustor pressure sensor (CIP1) and various far-field microphones are shown in figures 4 and 5. Aligned MSC and cross-spectra phase measurements between turbine exit pressure sensor (T551) and various far-field microphones are shown in figures

9 of 19

American Institute of Aeronautics and Astronautics 
6 and 7. Aligned MSC and cross-spectra phase measurements between turbine exit pressure sensor (T552) and various far-field microphones are shown in figures 8 and 9.

The magnitude-squared coherence function (MSC) plots will be discussed using logarithmic scales. Using logarithmic scales makes it easier to show that the analysis is based on MSC values above the threshold for unaligned signals. In addition to the MSC, also shown in Figs. 5, 7, 9, and 10 are MSC thresholds calculated from $\mathrm{Eq}(18)$.

The 95 percent threshold confidence interval based on $n_{s}=1120$ independent samples is $2.67 \times 10^{-3}$. However, the spectra are calculated using a 50 percent overlap to reduce the variance and the 95 percent threshold confidence interval based on $n_{s}=2240$ samples is $1.337 \times 10^{-3}$. These thresholds are shown in Figs. 4, 7, 9, and 10. These indicators show the MSC function is reliable up to about $400 \mathrm{~Hz}$ even though the pattern matching method used only cross-spectrum phase information from 16 to $208 \mathrm{~Hz}$. However, MSC function is above 0.1 in a region from $16-208 \mathrm{~Hz}$.

The slope change in Fig. 4 above $200 \mathrm{~Hz}$ indicates that indirect combustion noise is important below 200 $\mathrm{Hz}$ and direct combustion noise is important above $200 \mathrm{~Hz}$.

\section{Core noise source}

In order to evaluate the relative strength of indirect combustion noise and the turbine exit coherent broadband noise which contains both indirect and direct combustion noise a comparison of aligned MSC between combustor pressure sensor (CIP1) and far-field microphone at 130 degrees with aligned MSC between turbine pressure sensors (T551) and (T552) and far-field microphones at 48 percent maximum power is shown in Fig 10 using both linear and logarithmic scales. Fig $10 \mathrm{~b}$ also shows MSC threshold plots for $n_{s}=1120$ and $n_{s}=2240$ samples. Figure 10 shows both indirect and direct combustion noise are important below $400 \mathrm{~Hz}$.

\section{Concluding remarks}

A phase-based adaptive estimation pattern matching approach has been developed to estimate the post combustion (post-flame) time delay in the combustor from cross-spectral phase measurements made using a combustor pressure sensor, two turbine exit pressure sensors, and far-field microphones by estimating the propagation time to the far field for the indirect combustion noise and the turbine exit coherent broadband noise and taking the difference. The procedure accurately estimates the post-combustion/post-flame residence time in the combustor. It is the presence of indirect combustion noise that makes the procedure feasible. While the method has general application, the method discussed works well in this particular case due to the nature of the data set. The number of independent averages is chosen to be large $\left(n_{s} \approx 1120\right)$ and the magnitude-squared coherence (MSC) is over 0.1 up to $208 \mathrm{~Hz}$ making the standard deviation of the cross-spectra phase small below $200 \mathrm{~Hz}$. An accurate algorithm for calculating the post-combustion (post-flame) residence time in the combustor might be important in understanding the formation of thermal $\mathrm{NO}_{x}$ in the combustor and verifying new and innovative combustor designs based on computational tools. Calculating the aligned MSC is a necessary step in applying array processing methods to the MSC data set. In general, the adaptive estimation technique developed herein is an effective tool to determine time delays between broadband coherent signals in noisy environments such as exist in real aircraft engines.

\section{Acknowledgments}

This work was carried out under the NASA Fundamental Aeronautics Program, Fixed Wing Project, Quiet Performance Subproject.

10 of 19 


\section{References}

${ }^{I}$ Williams, J. E. F, and Howe, M. S., "The generation of sound by density inhomogeneities in low Mach number nozzle flows," J. Fluid Mech., Vol. 70 Part 3, 1970, pp. 605-622.

${ }^{2}$ Pickett, G. F., "Core Engine Noise Due to Temperature Fluctuating Through Turbine Blade Rows," Tech. Rep. AIAA-75-528, AIAA, 1975.

${ }^{3} \mathrm{Marble}_{z}$ F. E. and Candel, S. M., "Acoustic disturbance from gas non-uniformities convected through a nozzle," Journal of Sound and Vibration, Vol. 55 No. 2, 1977, pp. 225-243.

${ }^{4}$ Cumpsty, N. A. and Marble, F. E., "The Interaction of Entropy Fluctuations With Turbine Blade Rows; A Mechanism of Turbojet Noise." Proc. R. Soc. Lond. A., Vol. 357, 1977, pp. 323-344.

${ }^{5}$ Cumpsty, N. A. and Marble, F., "Core Noise from Gas Turbine Exhausts," Journal of Sound and Vibration, Vol. 54 No. 2, 1977, pp. 297-309.

${ }^{6}$ Cumpsty, N. A., Jet Engine Combustion Noise; Pressure, Entropy and Vorticity Perturbations Produced by Unsteady Combustion or Heat Addition," Journal of Sound and Vibration, Vol. 66 No. 4, 1979, pp. 527-544.

${ }^{7}$ Gliebe, P., Mani, R., Shin, H., Mitchell, B., Ashford, G., Salamah, S., and Connell, S., "Acoustic Prediction Codes,"

Tech. Rep. NASA CR-2000-210244, R99AEB169, General Electric Aircraft Engines, August 2000.

${ }^{8}$ Mani, R., "Issues in Combustion Noise," Annual Research Briefs 2007, Center for Turbulence

Research, Department of Mechanical Engineering , Stanford University Stanford, Ca, 94305,USA, 2007, http://ctr.stanford, edu/ResBriefs07/22_mani_pp255_264.pdf

${ }^{9}$ Bodony, D. J., "Scattering of an entropy disturbance into sound by a symmetric thin body" Physics of Fluids, Vol. 21,2009 , pp. 096101-1-10.

${ }^{10}$ Leyko, M., Nicoud, F., and Poinsot, T., "Comparison of Direct and Indirect Combustion Noise Mechanisms in a Model Combustor," AIAA Journal, Vol. No., November 2009, pp. 2709-2716.

${ }^{11}$ Miles, J. H., Wasserbauer, C. A., and Krejsa, E. A., "Cross Spectra Between Temperature and Pressure in a Constant Area Duct Downstream of a Combustor," AIAA-83-0762, NASA TM-83351, 1983.

${ }^{12}$ Miles, J. H., Wasserbauer, C. A., and Krejsa, E., "Cross spectra between pressure and temperature in a constantarea duct downstream of a hydrogen-fueled combustor," Tech. Rep. NASA-TM-83463, NASA Glenn Research Center, 1983.

${ }^{13}$ Miles, J. H., "Time Delay Analysis of Turbofan Engine Direct and Indirect Combustion Noise Sources," Journal of Propulsion and Power, Vol. 25 No. 1, January-February 2009, pp. 218-227.

${ }^{14}$ Miles, J. H., "Core Noise Diagnostics of Turbofan Engine Noise Using Correlation and Coherence Functions," Journal of Propulsion and Power, Vol. 26 No, 2, March/April 2010, pp. 303-316.

${ }^{15}$ Miles, J. H., "Separating Direct and Indirect Turbofan Engine Combustion Noise Using the Correlation Function," Journal of Propulsion and Pover, Vol, 26 No. 5, September-October 2010, pp. 1144-1152.

${ }^{16}$ Weir(editor), D. S., "Engine Validation of Noise \& Emission Reduction Technology Phase 1," Tech. Rep. NASA/CR2008-215225, Honeywell, May 2008.

${ }^{17}$ Etter, D. M. and Stearns, S. D., "Adaptive Estimation of Time Delays in Sampled Data Systems," IEEE Transactions on Acoustics, Speech, and Signal Processing, Vol. ASSP-29 3, June 1981, pp. 582-587.

${ }^{18}$ Carter, G. C., "Time Delay Estimation for Passive Sonar Signal Processing," IEEE Transactions on Acoustics, Speech, and Signal Processing, Vol. ASSP-29 3, June 1981, pp. 463-470.

${ }^{19} \mathrm{Knapp}, \mathrm{C} . \mathrm{H}$. and Carter, G. C., "The generalized correlaton method for estimation of time delay," IEEE Trans. Acoust, Speech, Signal Processing, Vol. ASSP-24 No. 4, 1976, pp. 320-327.

${ }^{20}$ Azaria, M. and Hertz, D., "Time Delay Estimation by Generalized Cross Correlation Methods," IEEE Transactions on Acoustics, Speech, and Signal Processing, Vol. 32 2, April 1984, pp. 280-285.

${ }^{21}$ Jacovitti, G. and Scarano, G., "Discrete Time Techniques for Time Dealy Estimation," IEEE Transactions on signal processing, Vol. 41 No. 2, February 1993, pp. 525-533.

${ }^{22}$ Viola, F, and Walker, W. F., "A Comparison of the Performance of Time-Delay Estimators in Medical Ultrasound," IEEE Transactions on Ultrasonics, Ferroelctrics, and Frequency Control, Vol. 50 4, April 2003, pp. 392-401.

${ }^{23}$ Viola, F. and Walker, W. F., "A Spline-Based Algorithm for Continuous Time-Delay Estimation Using Sampled

Data," IEEE Transactions on Ultrasonics, Ferroelctrics, and Frequency Control, Vol. 52 1, January 2005, pp. 80-93.

${ }^{24}$ Piersol, A., "Time Delay Estimation Using Phase Data," IEEE Transactions on Acoustics, Speech, and Signal Processing, Vol. ASSP-29 No. 3, June 1981, pp. 471-477.

${ }^{25}$ Chan, Y. T., Hattin, R., and J.B.Plant, "The least squares estimation of time delay and its use in signal detection," IEEE Trans. Acoust. Speech, Signal Processing, Vol. ASSP-26 No. 3, June 1978, pp. 217-222.

${ }^{26}$ Zhen, Z. and Zi-qiang, H., "The Geneaalized Phase Spectrtm Method for Time Delay Estimation," Prloc. ICASSP'84,vol. 3,pp46.2/1-4,March, 1984.

${ }^{27}$ Daneti, M., "On using phase data information for pipeline leak location," Electrical and Electronics Engineers in Israel, 2008. IEEEI 2008. IEEE 25th Convention of, IEEE, 2008, pp. 494-498.

${ }^{28}$ Miles, J. H., "Spectral Separation of the Turbofan Engine Coherent Combustion Noise Component," AIAA-2008-50, January 2008, NASA TM-2008-215157-0,2008.

${ }^{29}$ Welch, P. D., "The Use of Fast Fourier Transform for the Estimation of Power Spectra: A Method Based on Time Averaging Over Short, Modified Periodograms," IEEE Transactions on audio and electroacoustics, Vol. AU-15 No.2, June 1967, pp. $70-73$.

${ }^{30}$ Stearns, S. D. and David, R. A., Signal Processing Algorithms Using Fortran and C, Prentice-Hall, Inc, 1993.

${ }^{31}$ Porat, B., Digital Processing of Random Signals, Dover Publications, NY, 1994,2008.

${ }^{32}$ Hayes, M. H., Statistical Digital Signal Processing and Modeling, John Wiley \& Sons, 1996.

11 of 19

American Institute of Aeronautics and Astronautics 
${ }^{33}$ Manolakis, D. G., Ingle, V. K., and Kogon, S. M., Statistical and Adaptive Signal Processing, Artech House, Boston, Massachusetts and London, United Kingdom, 2005.

${ }^{34}$ Bendat, J. S., "Statistical errors in measurement of coherence functions and input/output quantities," Journal of Sound and Vibration, Vol. 59 No. 3, 1978, pp. 405-421.

${ }^{35}$ Bendat, J. S. and Piersol, A. G., Engineering Applications of Correlation and Spectral Analysis, John Wiley \& Sons, 1980 , pages $175,167-172,215$.

${ }^{36}$ Miles, J. H., "Aligned and Unaligned Coherence: A New Diagnostic Tool," Tech. Rep. AIAA-2006-0010, AIAA, 2006, Presented at the 44th AIAA Aerospace Science Meeting, 9-12 Jan 2006 Reno Hilton Reno, Nevada, also NASA/TM2006-214112.

${ }^{37}$ Miles, J. H., "Estimation of signal coherence threshold and concealed spectral lines applied to detection of turbofan engine combustion noise," The Journal of the Acoustics Society of America, Vol. 129 No. 5, May 2011, pp. 3068-3081.

${ }^{38} \mathrm{Gibra}$, I. N., Probability and Statistical Inference for Scientists and Engineers, Prencice-Hall, Inc., 1973, page $412-415$

${ }^{39}$ Edwards, A. L., An Introduction to Linear Regression and Correlation, W. H. Freeman and Company, 1976, pages 24-31.

${ }^{40}$ Wackerly, D. D. and III, W. M., Mathematical Statistics with Applicatioins, Duxbury Pacific Grove, CA, 2002, pages 537-540.

${ }^{41}$ Chatterjee, S. and Hadi, A. S., Regressiion Analysis by Example (Fourth Edition), John Wiley \& Sons, 2006, pages

$29-30$

${ }^{42}$ Powell, M. J. D., "An Efficient Method of Finding the Minimum of a Function of Several Variables without Calculating Derivatives," Computer Journal, Vol. 7 No. 2, July 1964, pp. 155-162.

${ }^{43}$ Kuester, J. L. and Mize, J. H., Optimization Techniques with Fortran, McGraw-Hill, 1973.

${ }^{44}$ Fox, J., An $R$ and S-Plus Campanion to Applied Regression, Sage Publications, 2002, page 86-89.

${ }^{45}$ Fox, J. and Weisberg, S., An $R$ Campanion to Applied Regression, Sage Publications, 2011, page $107-111$.

${ }^{46}$ Miles, J. H., unpublished.

${ }^{47}$ Miles, J. H., "Separating Direct and Indirect Turbofan Engine Combustion Noise While Estimating the PostCombustion (Post-Flame) Residence Time Using the Correlation Function," Tech. Rep. NASA-TM-2011-216248, NASA, 2011. 
Table 1. Spectral estimate parameters.

\begin{tabular}{lr}
\hline \hline Parameter & Value \\
\hline Segment length, (data points per segment), $N$ & 4096 \\
Sample rate, $r_{s}$, samples $/ \mathrm{s}$ & 65536 \\
Segment length, $T_{d}=N / r_{s}, \mathrm{~s}$ & 0.062500 \\
Sampling interval, $\Delta t=1 / r_{s}, \mathrm{~s}$ & $1 / 65536$ \\
Bandwidth resolution, $B_{e}=\Delta f=1 / T_{d}=r_{s} / N, \mathrm{~Hz}$ & 16.0 \\
Upper frequency limit, $f_{u}=1 / 2 \Delta t=r_{s} / 2, \mathrm{~Hz}$ & 32768 \\
Number of frequencies, $L y=f_{c} / \Delta f=N / 2$ & 2048 \\
Number of independent samples, $n_{s}$ & $\approx 1120$ \\
Overlap & 0.50 \\
Sample length, $T_{\text {total }, \mathrm{s}}$ & $\approx 70$ \\
\hline \hline
\end{tabular}

Table 2. Post-combustion (post-flame) residence time in the combustor calculated from difference of propagation time to far-field microphones of indirect combustor noise signal and acoustic signal at 48 percent maximum power.

\begin{tabular}{|c|c|c|c|c|c|c|c|}
\hline $\begin{array}{l}\text { Far-field } \\
\text { Microphone }\end{array}$ & CIP1 & $\mathrm{T} 551$ & T552 & $\Delta_{T 551}^{C I P 1}$ & $\Delta_{T 552}^{C I P 1}$ & $\begin{array}{c}D_{k \ell}^{T 551} \\
\times 1000 / 2^{16}\end{array}$ & $\begin{array}{c}D_{k \ell}^{T 552} \\
\times 1000 / 2^{16}\end{array}$ \\
\hline at $100 \mathrm{ft}$ & counts & counts & counts & counts & counts & $\mathrm{ms}$ & $\mathrm{ms}$ \\
\hline $90^{\circ}$ & 6349 & 6099 & 6091 & 250 & 258 & 3.814697 & 3.936768 \\
\hline $110^{\circ}$ & 6098 & 5842 & 5836 & 256 & 262 & 3.906250 & 3.997803 \\
\hline $130^{\circ}$ & 5878 & 5619 & 5611 & 259 & 267 & 3.952026 & 4.074097 \\
\hline $160^{\circ}$ & 5609 & 5349 & 5345 & 260 & 264 & 3.967285 & 4.028320 \\
\hline mean $(\tau)$ & $3.960 \mathrm{~ms}$ & & & & & & \\
\hline standard deviation $(\tau)$ & $0.079 \mathrm{~ms}$ & & & & & & \\
\hline
\end{tabular}


Table 3. Post-combustion (post-flame) residence time in the combustor calculated from difference of propagation time to far-field microphones of indirect combustor noise signal and acoustic signal at 54 percent maximum power.

\begin{tabular}{|c|c|c|c|c|c|c|c|}
\hline \multirow{3}{*}{$\begin{array}{l}\text { Far-field } \\
\text { Microphone } \\
\text { at } 100 \mathrm{ft}\end{array}$} & \multirow[t]{2}{*}{ CIP1 } & \multirow[t]{2}{*}{ T551 } & \multirow[t]{2}{*}{ T552 } & \multirow[t]{2}{*}{$\Delta_{T 551}^{C I P 1}$} & \multirow[t]{2}{*}{$\Delta_{T 552}^{C I P 1}$} & \multirow{2}{*}{$\begin{array}{c}D_{k \ell}^{T 551} \\
\times 1000 / 2^{16}\end{array}$} & \multirow{3}{*}{$\begin{array}{c}D_{k \ell}^{T 552} \\
\times 1000 / 2^{16} \\
\mathrm{~ms}\end{array}$} \\
\hline & & & & & & & \\
\hline & counts & counts & counts & counts & counts & $\mathrm{ms}$ & \\
\hline $90^{\circ}$ & 6367 & 6131 & 6124 & 236 & 243 & 3.601074 & 3.707886 \\
\hline $110^{\circ}$ & 6112 & 5868 & 5859 & 244 & 253 & 3.723145 & 3.860474 \\
\hline $130^{\circ}$ & 5891 & 5640 & 5636 & 251 & 255 & 3.829956 & 3.890991 \\
\hline $160^{\circ}$ & 5620 & 5366 & 5368 & 254 & 252 & 3.875732 & 3.845215 \\
\hline mean $(\tau)$ & $3.792 \mathrm{~ms}$ & & & & & & \\
\hline standard deviation $(\tau)$ & $0.103 \mathrm{~ms}$ & & & & & & \\
\hline
\end{tabular}

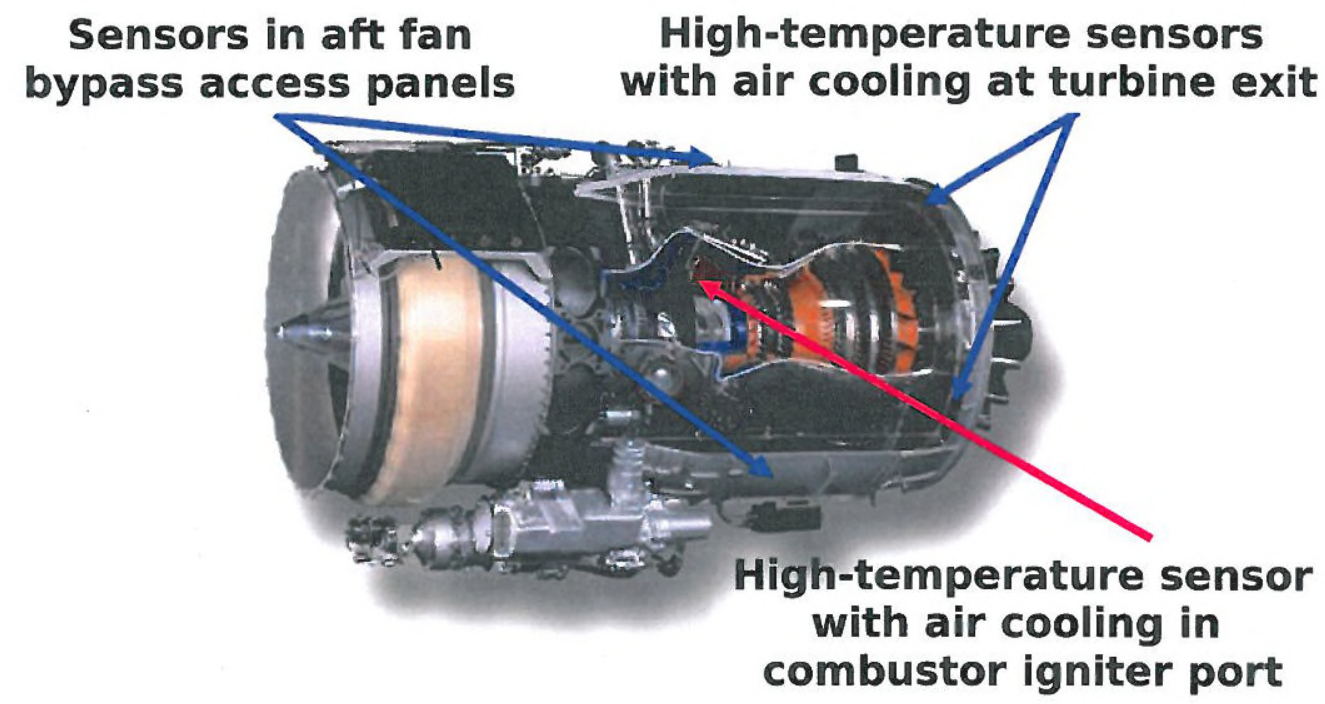

Figure 1. Honeywell TECH977 engine. 


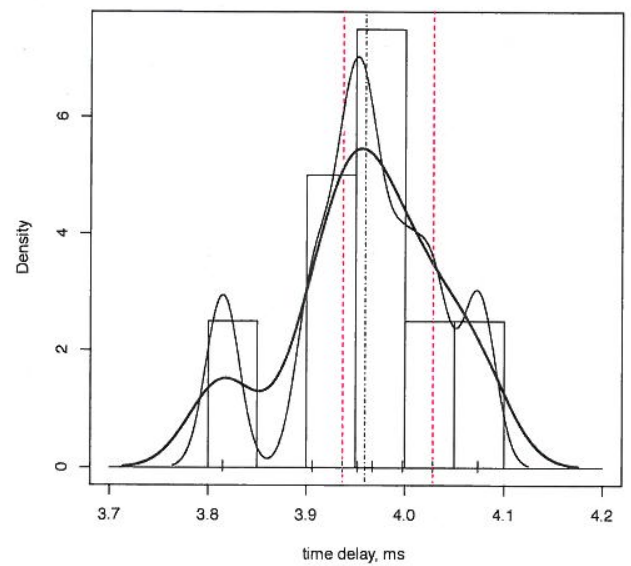

Figure 2. Histogram of post-combustion residence times in combustor in ms at 48 percent maximum power. Shown at the bottom of the plot are the location of the actual estimates as a scatter plot using a vertical bar $\mid$ as the plotting symbol.Also shown are $\tau_{\text {mean }}$ (blue dashed), $\tau_{C I P 1, T 551}$ (red dashed), and $\tau_{C I P 1, T 552}$ (red dashed).

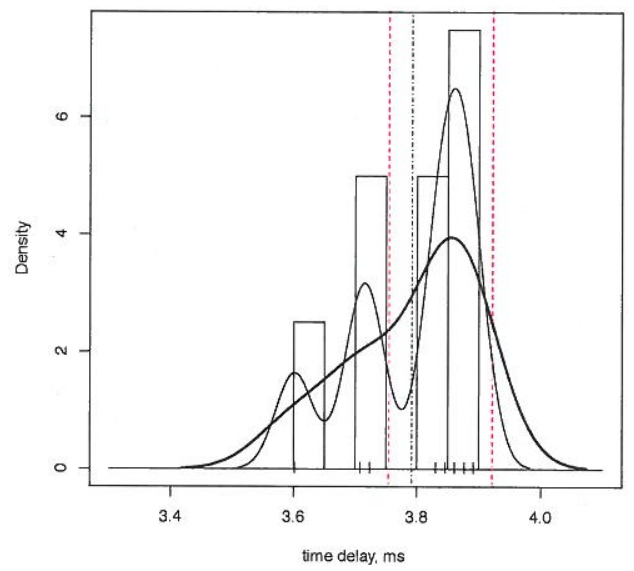

Figure 3. Histogram of post-combustion residence times in combustor in ms at 54 percent maximum power. Shown at the bottom of the plot are the location of the actual estimates as a scatter plot using a vertical bar $\mid$ as the plotting symbol.Also shown are $\tau_{\text {mean }}$ (blue dashed), $\tau_{C I P 1, T 551}$ (red dashed), and $\tau_{C I P 1, T 552}$ (red dashed).

15 of 19

American Institute of Aeronautics and Astronautics 


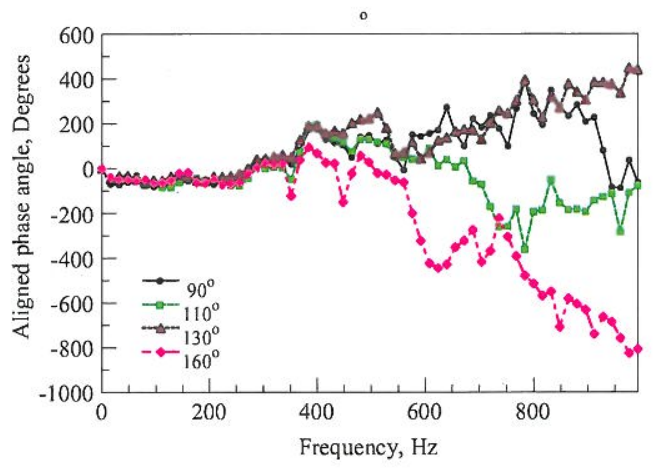

Figure 4. Aligned cross-spectra phase angle between combustor pressure sensor (CIP1) and far-field microphones at 48 percent maximum power.

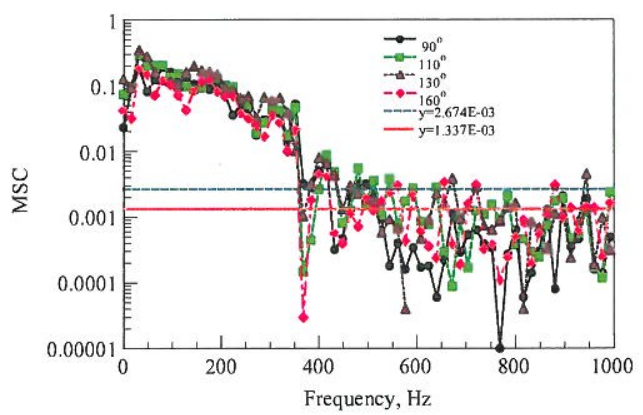

Figure 5. Aligned coherence between combustor pressure sensor (CIP1) and far-field microphones at 48 percent maximum power.

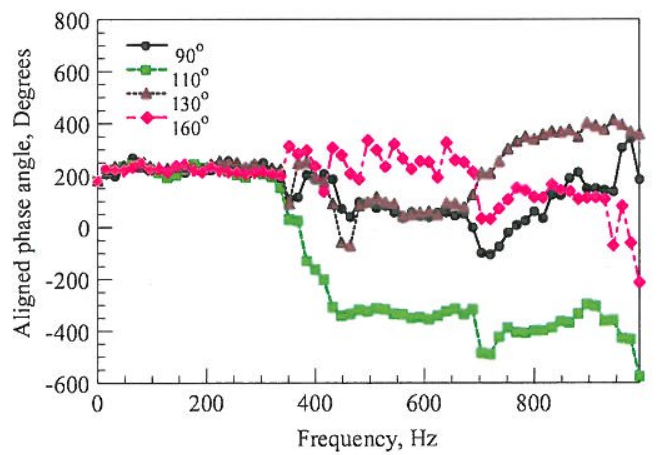

Figure 6. Aligned cross-spectra phase angle between turbine pressure sensor (T551) and far-field microphones at 48 percent maximum power. 


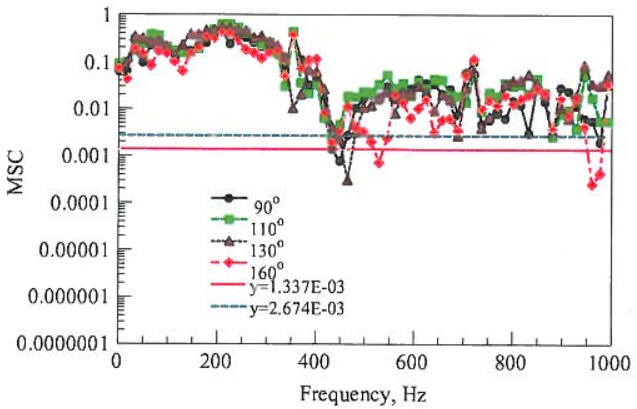

Figure 7. Aligned coherence between turbine pressure sensor (T551) and far-field microphones at 48 percent maximum power.

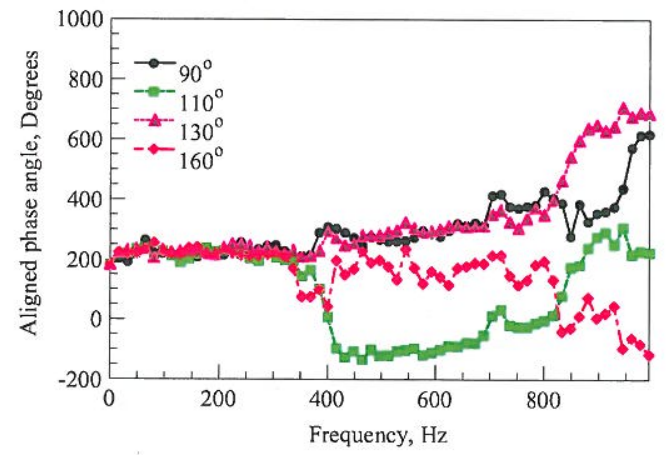

Figure 8. Aligned cross-spectra phase angle between turbine pressure sensor (T552) and far-field microphones at 48 percent maximum power.

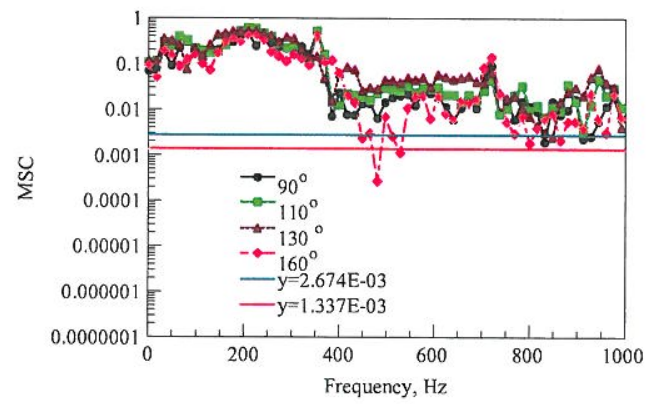

Figure 9. Aligned coherence between combustor pressure sensor (T552) and far-field microphones at 48 percent maximum power.

17 of 19

American Institute of Aeronautics and Astronautics 


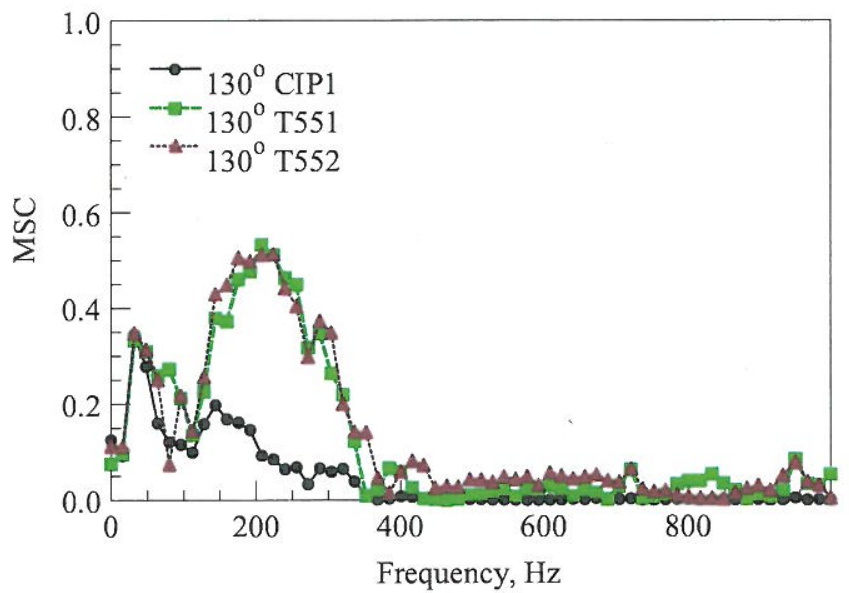

(a) Linear scale.

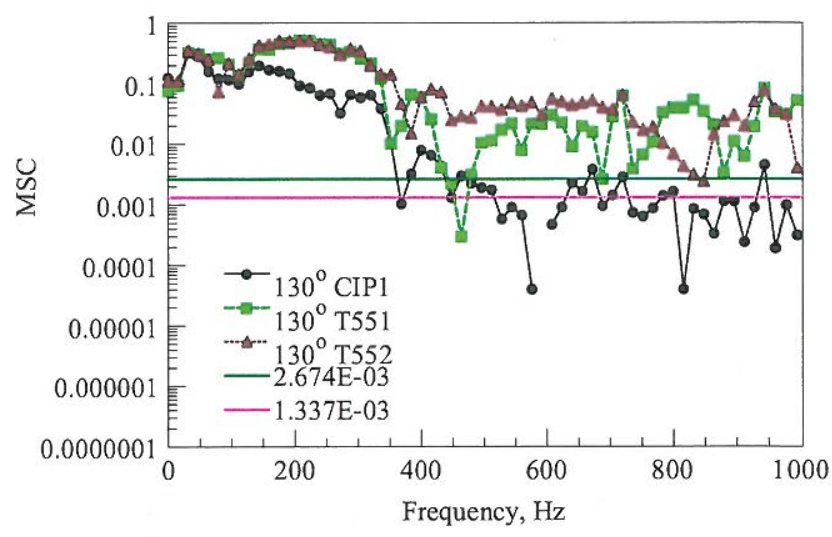

(b) Logarithmic scale

Figure 10. Comparison of aligned coherence between combustor pressure sensor (9) and far-field microphone at 130 degrees with aligned coherence between turbine pressure sensors (10) and (11) and far-field microphones at 48 percent maximum power. 